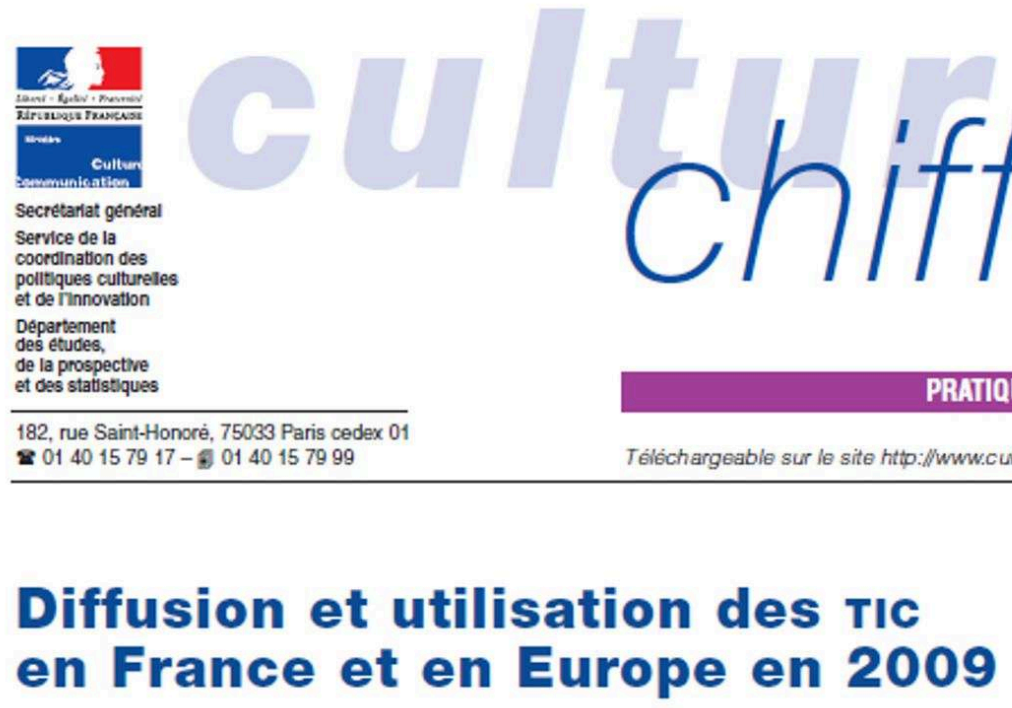

Valérie Deroin*

Spread and use of ICT in France and Europe in 2009

\section{L'ACCÈS DES MÉNAGES}

\section{AUX ÉQUIPEMENTS NUMÉRIQUES}

\section{L'équipement informatique}

Un Français ${ }^{1}$ sur deux utilise quotidiennement un ordinateur

Depuis une décennie, l'engouement pour les produits liés aux technologies de l'information et de la communication (TIC) n'a pas faibli en Europe. Le poids des dépenses informatiques représente $7 \%$ des dépenses culturelles et de loisirs des Français en 2008 et, depuis 1997, le poste « dépenses en informatique » est celui qui a le plus progressé dans le budget total des dépenses culturelles et de loisirs des ménages ( $+30 \%$ environ).

La pratique informatique confirme ce comportement: l'achat d'un ordinateur s'assortit le plus souvent d'un usage quotidien. De fait, les Français qui disposent d'un ordinateur sont de très réguliers utilisateurs : en $2009,56 \%$ en ont fait un usage quotidien au cours des trois derniers mois (tableau 1).

Néanmoins, $20 \%$ des Français déclarent encore ne jamais avoir utilisé d'ordinateur en 2009. Cette proportion atteint $26 \%$ pour les Européens des 27 États membres.

Tableau I - Fréquence d'utilisation numérique des particuliers, Union européenne des 27 et France, 2008-2009

\begin{tabular}{|c|c|c|c|c|}
\hline & \multicolumn{2}{|c|}{ Union européenne (27 pays) } & \multicolumn{2}{|c|}{ France } \\
\hline & 2008 & 2009 & 2008 & 2009 \\
\hline \multicolumn{5}{|l|}{$\begin{array}{l}\text { Part des individus ayant ut isé un ordinateur, } \\
\text { en moyenne, au cours des } 3 \text { derniers mois: }\end{array}$} \\
\hline chaque jour ou presque & 50 & 53 & 54 & 56 \\
\hline au moins une fois par semaine (mais pas tous les jours) & 12 & 11 & 13 & 13 \\
\hline au moins une fois par mois (mais pas chaque semaine) & 3 & 3 & 3 & 2 \\
\hline moins d'une fois par mois & 1 & 1 & 1 & 1 \\
\hline Part des individus n'ayant jamais utilisé un ordinateur au cours de l'annèe & 27 & 26 & 20 & 20 \\
\hline
\end{tabular}

* Attachée statisticienne de l'Insee, chargée d'analyse au peirs.

1. $N B$ : l'enquête européenne xcr couvre les ménages comptant au moins une personne âgŕe de 16 à 74 ans. 


\section{Diffusion et utilisation des TIC en France et en Europe en 2009}

Spread and use of ICT in France and Europe in 2009

\section{Valérie Deroin}

Éditeur : Département des études, de la prospective et des statistiques

Lieu d'édition : Paris

Année d'édition : 2010

Date de mise en ligne : 21 septembre 2015

Collection : Culture chiffres

ISBN électronique : 9782111398498

\section{Sbooks}

http://books.openedition.org

\section{Édition imprimée}

Date de publication : 1 mars 2010

Nombre de pages : 12

\section{Référence électronique}

DEROIN, Valérie. Diffusion et utilisation des TIC en France et en Europe en 2009. Nouvelle édition [en ligne]. Paris : Département des études, de la prospective et des statistiques, 2010 (généré le 25 avril 2021). Disponible sur Internet : <http://books.openedition.org/deps/558>. ISBN : 9782111398498. 

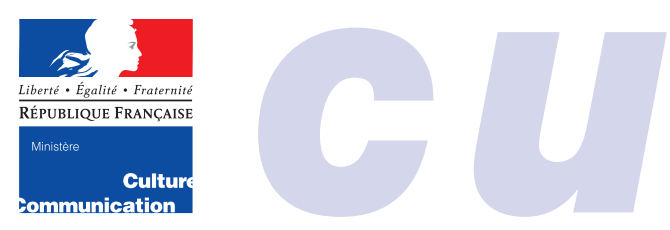

Secrétariat général

Service de la

coordination des

politiques culturelles

et de l'innovation

Département

des études,

de la prospective

et des statistiques

\title{
Diffusion et utilisation des TIC en France et en Europe en 2009
}

\author{
Valérie Deroin*
}

\section{Spread and use of ICT in France and Europe in 2009}

\section{L'ACCÈS DES MÉNAGES \\ AUX ÉQUIPEMENTS NUMÉRIQUES}

\section{L'équipement informatique}

\section{Un Français ${ }^{1}$ sur deux utilise quotidiennement un ordinateur}

Depuis une décennie, l'engouement pour les produits liés aux technologies de l'information et de la communication (TIC) n'a pas faibli en Europe. Le poids des dépenses informatiques représente $7 \%$ des dépenses culturelles et de loisirs des Français en 2008 et, depuis 1997, le poste «dépenses en informatique » est celui qui a le plus progressé dans le budget total des dépenses culturelles et de loisirs des ménages ( $+30 \%$ environ).

La pratique informatique confirme ce comportement: l'achat d'un ordinateur s'assortit le plus souvent d'un usage quotidien. De fait, les Français qui disposent d'un ordinateur sont de très réguliers utilisateurs : en 2009, $56 \%$ en ont fait un usage quotidien au cours des trois derniers mois (tableau 1).

Néanmoins, $20 \%$ des Français déclarent encore ne jamais avoir utilisé d'ordinateur en 2009. Cette proportion atteint $26 \%$ pour les Européens des 27 États membres.

\section{Tableau 1 - Fréquence d'utilisation numérique des particuliers,} Union européenne des 27 et France, 2008-2009

\begin{tabular}{|c|c|c|c|c|}
\hline & \multicolumn{2}{|c|}{ Union européenne (27 pays) } & \multicolumn{2}{|c|}{ France } \\
\hline & 2008 & 2009 & 2008 & 2009 \\
\hline \multicolumn{5}{|l|}{$\begin{array}{l}\text { Part des individus ayant utilisé un ordinateur, } \\
\text { en moyenne, au cours des } 3 \text { derniers mois: }\end{array}$} \\
\hline chaque jour ou presque & 50 & 53 & 54 & 56 \\
\hline au moins une fois par semaine (mais pas tous les jours) & 12 & 11 & 13 & 13 \\
\hline au moins une fois par mois (mais pas chaque semaine) & 3 & 3 & 3 & 2 \\
\hline moins d'une fois par mois & 1 & 1 & 1 & 1 \\
\hline Part des individus n'ayant jamais utilisé un ordinateur au cours de l'année & 27 & 26 & 20 & 20 \\
\hline
\end{tabular}

\footnotetext{
* Attachée statisticienne de l'Insee, chargée d'analyse au DEPS.

1. NB : l'enquête européenne ICT couvre les ménages comptant au moins une personne âgée de 16 à 74 ans.
} 
Tableau 2 - Fréquence d'utilisation numérique par région géographique en France, 2009

\begin{tabular}{|c|c|c|}
\hline & $\begin{array}{l}\text { Particuliers ayant } \\
\text { eu accès à l'internet } \\
\text { au moins une fois } \\
\text { par semaine, } \\
\text { en moyenne }\end{array}$ & $\begin{array}{c}\text { Particuliers } \\
\text { n'ayant jamais } \\
\text { utilisé } \\
\text { un ordinateur }\end{array}$ \\
\hline France & 65 & 20 \\
\hline Île-de-France & 70 & 15 \\
\hline Méditerranée & 65 & 19 \\
\hline Est & 62 & 19 \\
\hline Centre-Est & 61 & 26 \\
\hline Bassin parisien & 60 & 25 \\
\hline Sud-Ouest & 60 & 24 \\
\hline Ouest & 56 & 28 \\
\hline Nord-Pas-de-Calais & 55 & 31 \\
\hline \multicolumn{3}{|c|}{$\begin{array}{l}\text { 1. Le découpage géographique européen de la nomenclature des unités ter- } \\
\text { ritoriales statistiques de niveau } 1 \text { (NUTS 1) correspond à la zone d'études et } \\
\text { d'aménagement du territoire (ZEAT) française. Le Bassin parisien comprend } \\
\text { les régions suivantes : la Bourgogne, le Centre, la Champagne-Ardenne, les } \\
\text { Basse et Haute-Normandie, la Picardie. L'Est regroupe l'Alsace, la Franche- } \\
\text { Comté, la Lorraine. L'Ouest: la Bretagne, les Pays de la Loire et le Poitou- } \\
\text { Charentes. Le Sud-Ouest : l'Aquitaine, le Limousin et Midi-Pyrénées. Le } \\
\text { Centre-Est: l'Auvergne et Rhône-Alpes. La Méditerranée: le Languedo-- } \\
\text { Roussillon, la Provence-Alpes-Côte d'Azur et la Corse. L'Île-de-France et le } \\
\text { Nord-Pas-de-Calais correspondent aux deux régions administratives fran- } \\
\text { çaises. }\end{array}$} \\
\hline
\end{tabular}

Source : Eurostat, enquête Technologie de l'information et de la communication/DEPS, 2010

\section{Graphique 1 - Utilisation quotidienne \\ d'un ordinateur au cours des \\ 3 derniers mois par tranche d'âge, Union européenne des 27 et France}

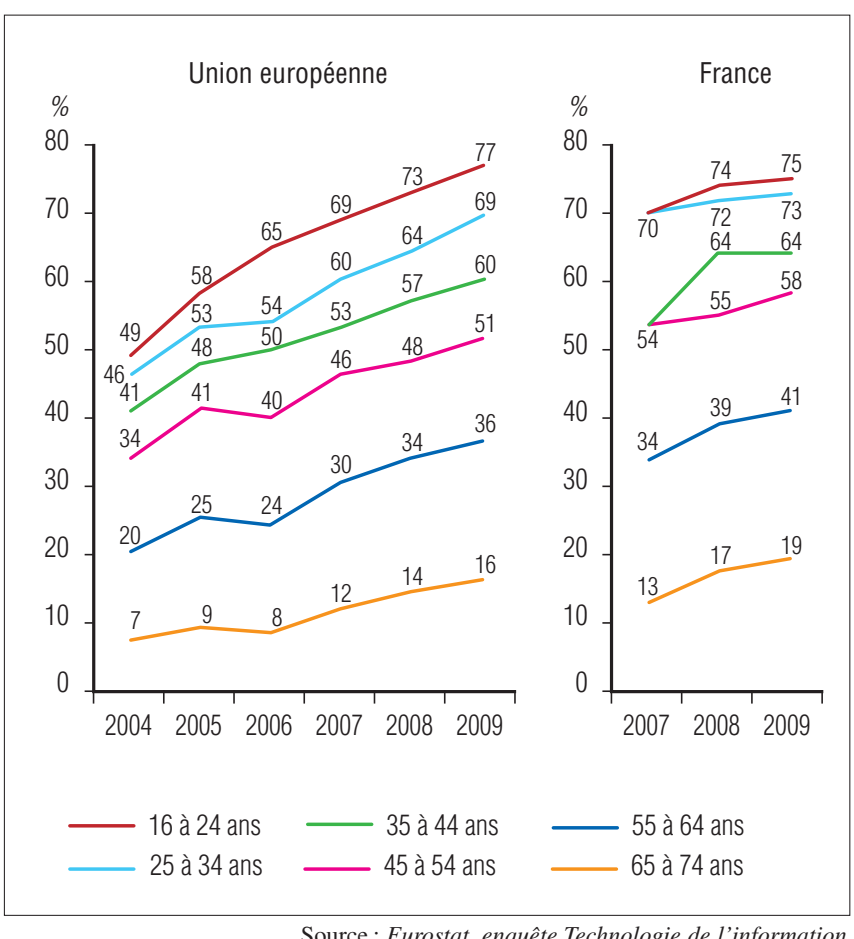

Source : Eurostat, enquête Technologie de l'information et de la communication/DEPS, 2010
En France, les écarts régionaux relatifs à l'utilisation de l'ordinateur et aux pratiques numériques sont importants, en particulier entre l'Île-de-France, le Nord et l'Ouest: si $15 \%$ des Franciliens affirment ne pas avoir utilisé d'ordinateur en 2009 , cette proportion concerne près d'un tiers (31\%) des particuliers résidant dans le Nord-Pas-de-Calais (tableau 2). Les régions dans lesquelles la proportion de non-utilisateurs d'ordinateur est la plus forte sont aussi celles dans lesquelles les particuliers se connectent le moins à l'internet. $65 \%$ des Français ont eu accès à l'internet une fois par semaine en moyenne au cours des trois derniers mois. La proportion est de $55 \%$ environ parmi les habitants du Nord-Pas-de-Calais, de l'Ouest (Bretagne, Pays de la Loire et Poitou-Charentes), et de $70 \%$ pour les Franciliens.

L'âge et le sexe restent deux critères discriminants dans l'usage des nouvelles technologies. Si l'utilisation quotidienne de l'ordinateur est en hausse, quel que soit l'âge, depuis 2006, les utilisateurs les plus assidus se comptent parmi les jeunes: l'écart d'utilisation quotidienne d'un ordinateur entre les 16-24 ans et les 55-64 ans est de 30 points environ. Ainsi la part d'utilisateurs quotidiens parmi la population française âgée de 16 à 24 ans est de $75 \%$ contre $41 \%$ pour les 55-64 ans - respectivement $77 \%$ et $36 \%$ dans l'Union européenne des 27 (graphique 1).

Par ailleurs, les jeunes hommes français âgés de 16 à 24 ans sont les plus grands utilisateurs d'ordinateur, $82 \%$ d'entre eux se servent chaque jour ou presque d'un ordinateur contre $68 \%$ des jeunes Françaises du même âge. Il en est de même parmi les tranches d'âge des aînés : les femmes âgées de 55 à 74 ans sont moins souvent devant un ordinateur que les hommes du même âge : $29 \%$ d'entre elles utilisaient un ordinateur chaque jour ou presque contre $36 \%$ d'hommes en 2009.

\section{L'accès à l'internet : six ménages sur dix accèdent à l'internet en 2009}

En France, le taux d'accès à l'internet se stabilise à $63 \%$ en 2009. Situé au $13^{\mathrm{e}}$ rang, au même niveau d'accès et d'usage que l'Estonie, le taux français reste proche de la moyenne européenne, égale à $65 \%$.

La moyenne européenne masque de fortes disparités parmi les 27 États membres : quand 8 à 9 ménages sur 10 sont raccordés à l'internet dans les pays nordiques (PaysBas, Luxembourg, Suède, Danemark), 3 à 4 ménages seulement le sont en Grèce, Roumanie ou Bulgarie (tableau 3 et carte 1).

\section{L'accès au haut débit des ménages se stabilise}

En France, plus d'un ménage sur deux (57\%) dispose dorénavant d'un accès au haut débit («à large bande »). Cette proportion est conforme à la moyenne européenne : $56 \%$ (graphique 2). Toutefois, l'accès au haut débit des ménages n'a pas encore suivi la généralisation opérée dans le monde de l'entreprise (92\% des entreprises françaises, 
Tableau 3 - Évolution du taux d'accès à l'internet des ménages en Union européenne de 2002 à 2009

20022003200420052006200720082009

\begin{tabular}{lrrrrrrrr} 
Union européenne & nd & nd & 41 & 48 & 49 & 54 & 60 & 65 \\
Pays-Bas & 58 & 61 & 65 & 78 & 80 & 83 & 86 & 90 \\
Luxembourg & 40 & 45 & 59 & 65 & 70 & 75 & 80 & 87 \\
Suède & nd & nd & nd & 73 & 77 & 79 & 84 & 86 \\
Danemark & 56 & 64 & 69 & 75 & 79 & 78 & 82 & 83 \\
Allemagne & 46 & 54 & 60 & 62 & 67 & 71 & 75 & 79 \\
Finlande & 44 & 47 & 51 & 54 & 65 & 69 & 72 & 78 \\
Royaume-Uni & 50 & 55 & 56 & 60 & 63 & 67 & 71 & 77 \\
Autriche & 33 & 37 & 45 & 47 & 52 & 60 & 69 & 70 \\
Belgique & nd & nd & nd & 50 & 54 & 60 & 64 & 67 \\
Irlande & nd & 36 & 40 & 47 & 50 & 57 & 63 & 67 \\
Malte & nd & nd & nd & 41 & 53 & 54 & 59 & 64 \\
Slovénie & nd & nd & 47 & 48 & 54 & 58 & 59 & 64 \\
Estonie & nd & nd & 31 & 39 & 46 & 53 & 58 & 63 \\
France & 23 & 31 & 34 & nd & 41 & 49 & 62 & 63 \\
Slovaquie & nd & nd & 23 & 23 & 27 & 46 & 58 & 62 \\
Lituanie & 4 & 6 & 12 & 16 & 35 & 44 & 51 & 60 \\
Pologne & 11 & 14 & 26 & 30 & 36 & 41 & 48 & 59 \\
Lettonie & 3 & nd & 15 & 31 & 42 & 51 & 53 & 58 \\
Hongrie & nd & nd & 14 & 22 & 32 & 38 & 48 & 55 \\
République tchèque & nd & 15 & 19 & 19 & 29 & 35 & 46 & 54 \\
Espagne & nd & 28 & 34 & 36 & 39 & 45 & 51 & 54 \\
Chypre & 24 & 29 & 53 & 32 & 37 & 39 & 43 & 53 \\
Italie & 34 & 32 & 34 & 39 & 40 & 43 & 47 & 53 \\
Portugal & 15 & 22 & 26 & 31 & 35 & 40 & 46 & 48 \\
Grèce & 12 & 16 & 17 & 22 & 23 & 25 & 31 & 38 \\
Roumanie & nd & nd & 6 & nd & 14 & 22 & 30 & 38 \\
Bulgarie & nd & nd & 10 & nd & 17 & 19 & 25 & 30 \\
\hline Croatie & nd & nd & nd & nd & nd & 41 & 45 & 50 \\
Serbie & nd & nd & nd & nd & nd & 26 & nd & 37 \\
Macédoine & nd & nd & 11 & nd & 14 & nd & 29 & 42 \\
Turquie & nd & nd & 7 & 8 & nd & 20 & nd & nd \\
\hline Islande & nd & nd & 81 & 84 & 83 & 84 & 88 & 90 \\
Norvège & nd & 60 & 60 & 64 & 69 & 78 & 84 & 86 \\
\hline nd: non disponible & & & & & & & & \\
& & & & & & & & \\
\hline
\end{tabular}

Source : Eurostat, enquête Technologie de l'information et de la communication/DEPs, 2010
Carte 1 - Taux d'accès à l'internet des ménages, 27 pays de l'Union européenne, 2002-2009

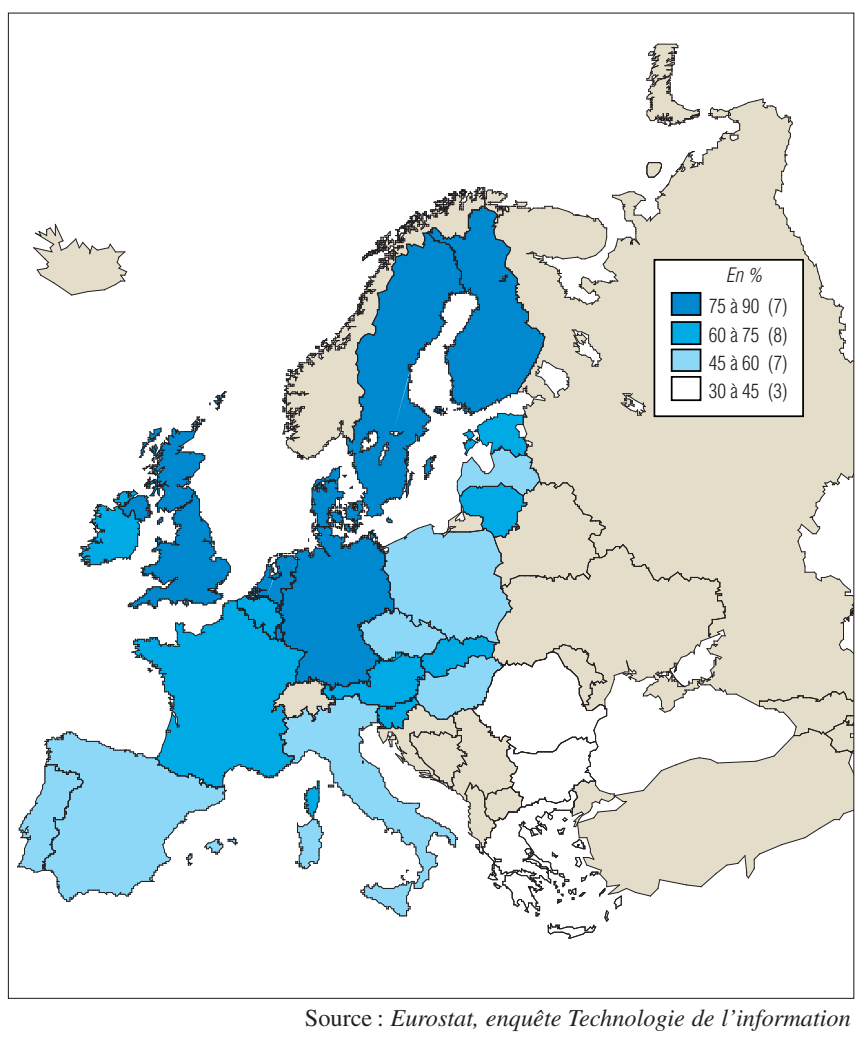

et de la communication/DEPS, 2010

toutes tailles confondues, contre $82 \%$ des entreprises à l'échelon européen).

Entre 2008 et 2009, la progression des connexions à haut débit des ménages est de $+14 \%$ dans l'Europe des 27, mais les évolutions restent inégales selon les pays. La progression la plus forte est réalisée par la Roumanie qui, bien qu'affichant le taux européen le plus bas de connexions à haut débit des ménages, multiplie presque par $2(+84 \%)$ ce taux de connexion, avec près d'un quart des ménages

Graphique 2 - Taux d'accès au haut débit des ménages, 27 pays de l'Union européenne, 2009

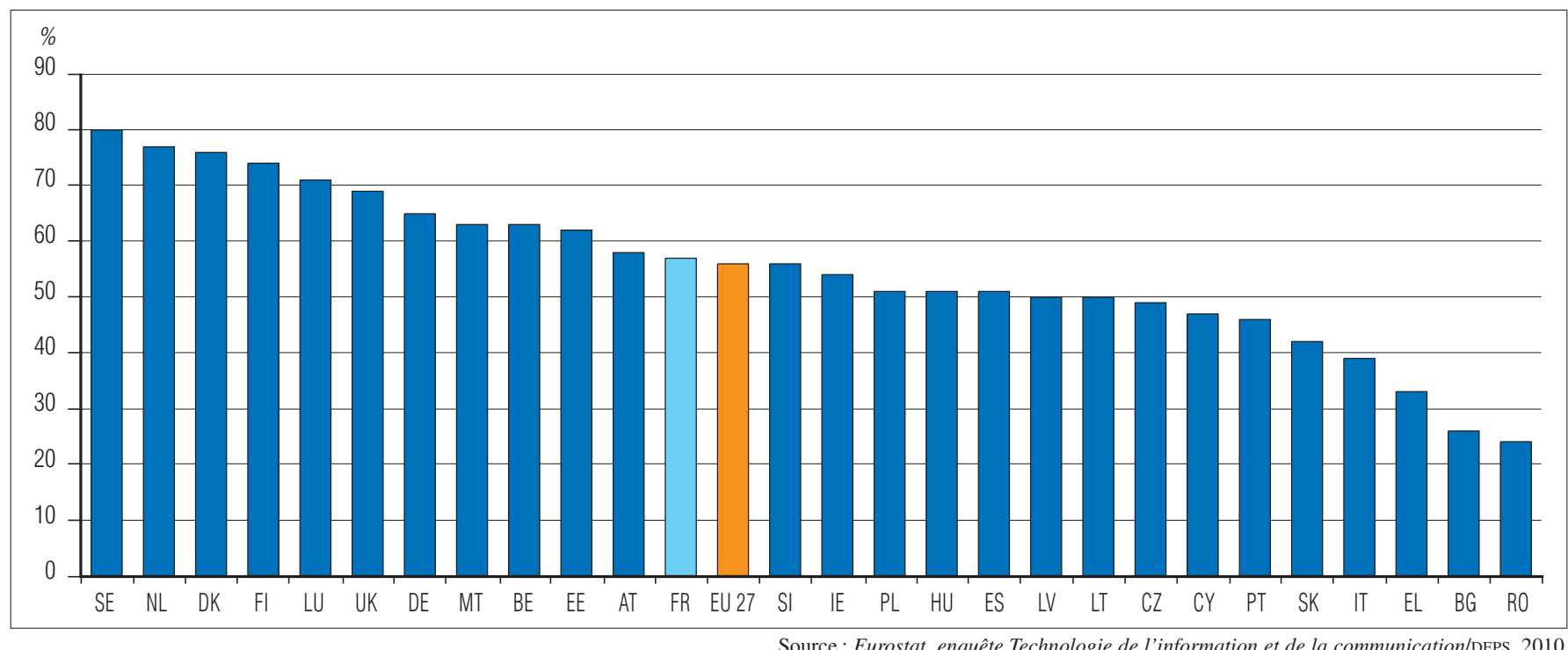


Graphique 3 - Taux d'accès à l'internet et au haut débit des ménages en France, 2006-2009

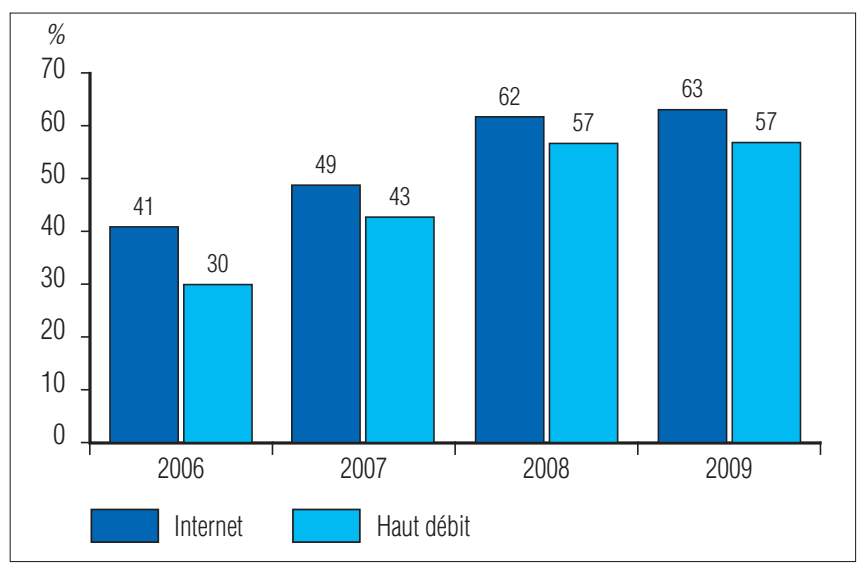

Source : Eurostat, enquête Technologie de l'information et de la communication/DEPS, 2010

(24\%) accédant à l'internet à haut débit. La France avait réalisé cette avancée entre 2006 et 2008, en développant de $38 \%$ en moyenne par an l'accès au haut débit des ménages (graphique 3).

\section{L'ordinateur reste le dispositif préféré pour accéder à l'internet}

Les Français comme les Européens se connectent à l'internet principalement depuis leur domicile (respectivement $60 \%$ et $58 \%$ en 2009). Néanmoins, un peu plus d'un quart des particuliers $(27 \%)$, en France comme en Europe, déclarent avoir aussi accédé à l'internet sur leur lieu de travail au cours des trois derniers mois.

En termes de dispositif d'accès à l'internet depuis le domicile, en 2009, les ménages français utilisent avant tout un ordinateur personnel (61\%). Au domicile, les nouveaux dispositifs d'accès sont encore peu répandus : $4 \%$ seulement par l'intermédiaire du poste de télévision. Les autres dispositifs nomades comme le téléphone mobile représentent $12 \%$ et l'ordinateur de poche $2 \%$ des moyens de connexion.

Entre 2008 et 2009, la tendance française est à la baisse et se positionne légèrement en deçà de la moyenne européenne : l'ordinateur personnel comme unique moyen de connexion à l'internet depuis le domicile concerne $44 \%$ des ménages français contre $49 \%$ des Européens (tableau 4).

Les pratiques de l'internet mobile s'intensifient néanmoins : l'utilisation de la téléphonie mobile et des ordinateurs portables comme dispositifs d'accès à l'internet, hors domicile, a progressé d'environ $30 \%$ dans l'ensemble des 27 États membres entre 2008 et 2009. Ainsi, les Européens sont $4 \%$ en 2009 à choisir d'utiliser un téléphone portable via le réseau UMTS $(3 \mathrm{G})$ pour se connecter à l'internet hors de leur domicile ou de leur lieu de travail, et $17 \%$ un ordinateur portable relié à un réseau sans fil (graphique 4).

Le Nord de l'Europe est en avance pour l'utilisation de ces outils : le Luxembourg se distingue très fortement par l'utilisation de l'ordinateur portable (44\%), suivi par le Danemark (31\%), la Suède (29\%), l'Estonie (28\%) et l'Irlande $(27 \%)$. La moyenne française est inférieure à celle des 27 États membres avec $14 \%$ de connexions par l'intermédiaire d'un ordinateur portable hors domicile ou bureau.

C'est aussi dans les pays du Nord que la téléphonie mobile $3 \mathrm{G}$ est la plus pratiquée ; elle a réalisé la plus forte progression au Luxembourg depuis 2008 et concerne $11 \%$ des connexions. La Suède (14\%) et le Danemark (10\%) restent en tête de cette pratique, accompagnés de pays du Centre et du Sud de l'Europe : Slovaquie (10\%), Slovénie (9\%) et Espagne (9\%). La France, bien qu'ayant doublé son taux de connexion par téléphonie mobile, est bien en deçà, avec $2 \%$ des connexions réalisées par l'intermédiaire de la téléphonie mobile.

Tableau 4 - Équipement numérique et accès des ménages à l'internet, Union européenne et France, 2008-2009

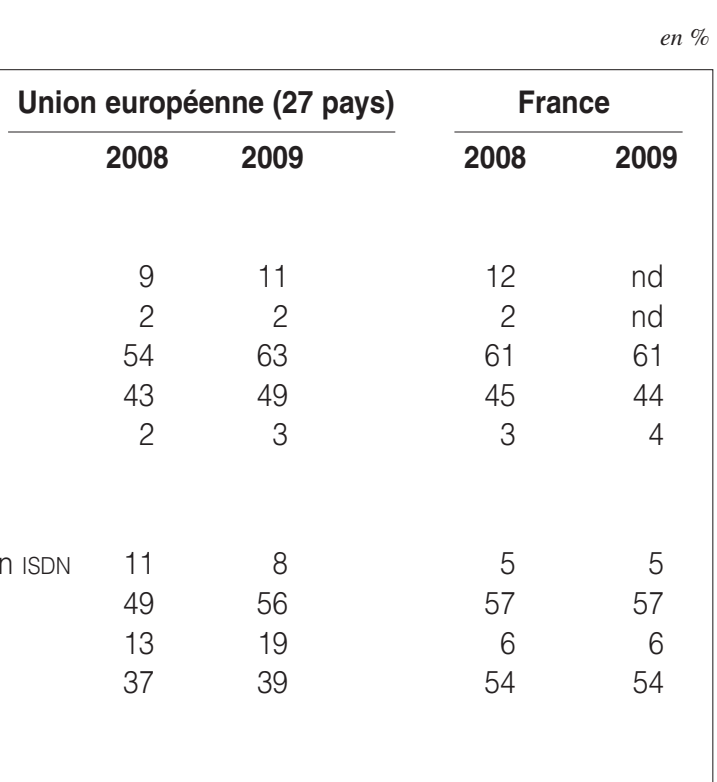

Source : Eurostat, enquête Technologie de l'information et de la communication/DEPS, 2010 
Graphique 4-Dispositifs d'accès mobile à l'internet, 27 pays de l'Union européenne, 2009

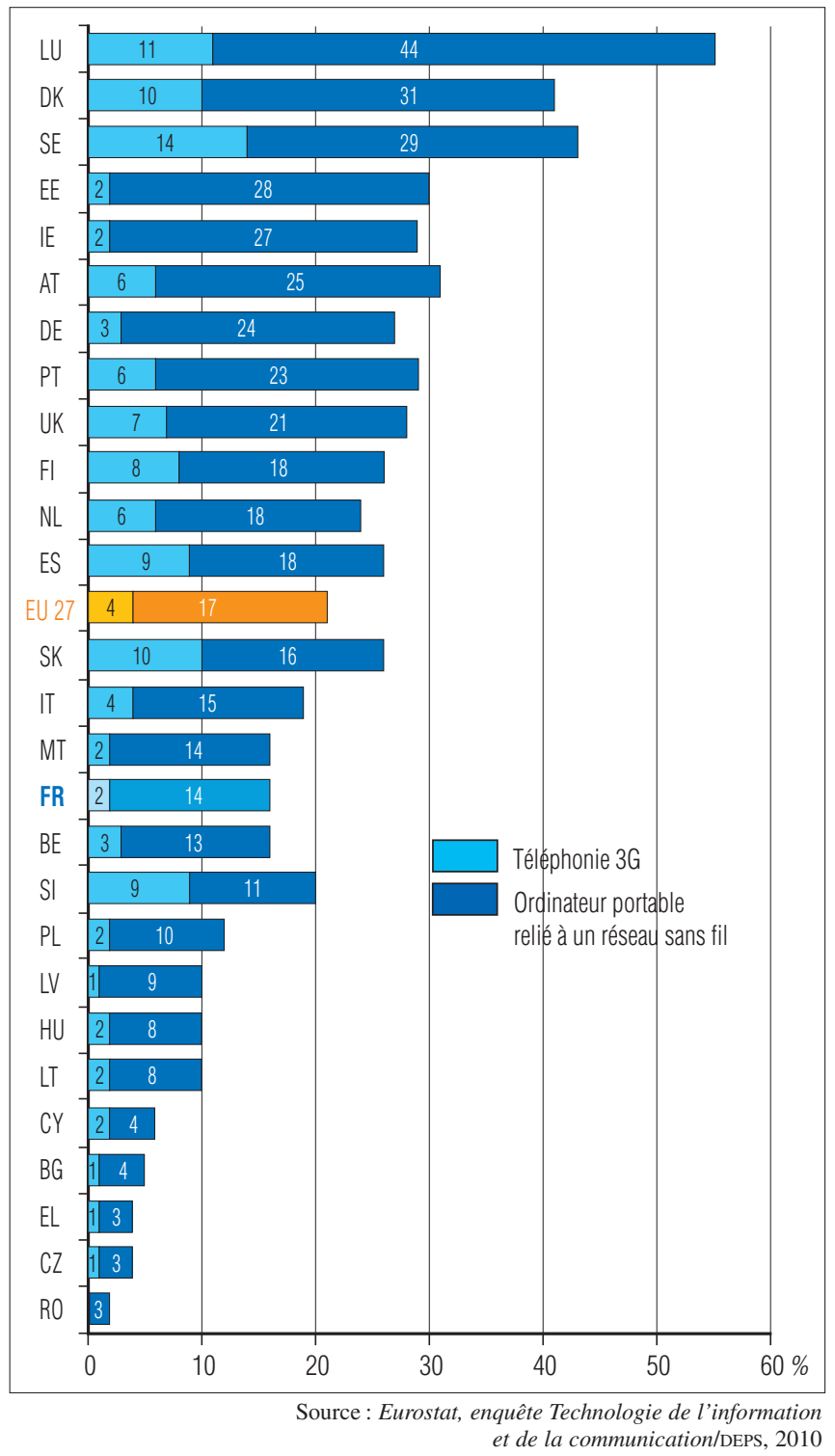

\section{LES USAGES NUMÉRIQUES}

\section{L'internet :}

\section{un outil de communication et de loisirs}

En 2009, la communication reste la principale activité des Français et des Européens : 6 Français sur 10 utilisent l'internet pour envoyer et lire des messages. Par ailleurs, 4 Français sur 10 recourent à des services bancaires en ligne (tableau 5).

Concernant les activités de loisirs, la recherche d'informations liées aux voyages et à l'hébergement occupe une place importante (37\%), suivie par le téléchargement en flux (streaming) d'émissions radiophoniques ou télévisuelles $(25 \%)$ et la lecture de la presse $(24 \%)$. L'analyse de l'évolution des activités pratiquées sur l'internet entre 2006 et 2009 suggère un élargissement des usages vers les services, notamment culturels et d'information (graphique 5) : progression des téléchargements (jeux, images,

\section{Graphique 5 - Activités des particuliers sur l'internet, Union européenne des 27 et France, 2006-2009}

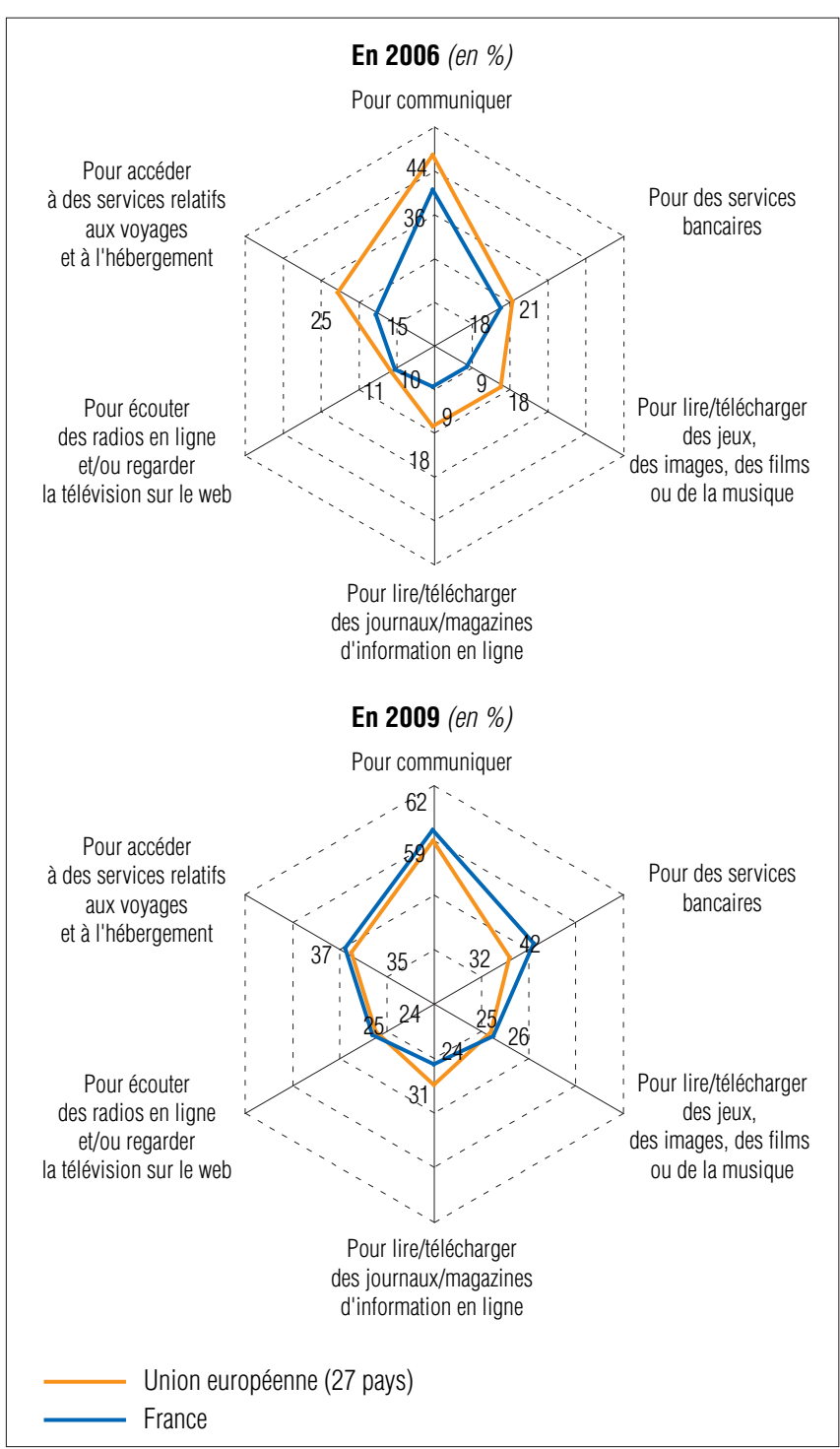

Source : Eurostat, enquête Technologie de l'information et de la communication/DEPS, 2010 
Tableau 5 - Activités des particuliers sur l'internet au cours des 3 derniers mois,

\begin{tabular}{|c|c|c|}
\hline & Union européenne (27 pays) & France \\
\hline $\begin{array}{l}\text { Activités de loisirs et informatiques } \\
\text { accéder à des services relatifs aux voyages et à l'hébergement } \\
\text { télécharger/écouter/jouer à/regarder de la musique, des films et des jeux } \\
\text { lire/télécharger des journaux/magazines d'information en ligne } \\
\text { écouter la radio, regarder la télévision en ligne } \\
\text { télécharger des logiciels }\end{array}$ & $\begin{array}{l}35 \\
\text { nd } \\
31 \\
24 \\
22\end{array}$ & $\begin{array}{l}37 \\
\text { nd } \\
24 \\
25 \\
19\end{array}$ \\
\hline $\begin{array}{l}\text { Activités de communication } \\
\text { communiquer } \\
\text { envoyer/recevoir des messages électroniques } \\
\text { publier un contenu créé par soi-même (texte, images, photos, vidéos, musi } \\
\quad \text { sur un site web en vue de le partager }\end{array}$ & $\begin{array}{l}59 \\
57\end{array}$ & $\begin{array}{l}62 \\
60\end{array}$ \\
\hline $\begin{array}{l}\text { Activités administratives et bancaires } \\
\text { contacter les pouvoirs publics } \\
\text { obtenir des informations à partir de sites web des pouvoirs publics } \\
\text { utiliser des services bancaires sur l'internet }\end{array}$ & $\begin{array}{l}29 \\
26 \\
32\end{array}$ & $\begin{array}{l}\text { nd } \\
\text { nd } \\
42\end{array}$ \\
\hline $\begin{array}{l}\text { Activités de formation et d'emploi } \\
\text { rechercher un emploi ou envoyer un acte de candidature } \\
\text { avoir des activités éducatives formalisées (école, université, etc.) } \\
\text { se former spécifiquement à des possibilités d'emploi }\end{array}$ & $\begin{array}{l}15 \\
\text { nd } \\
\text { nd }\end{array}$ & $\begin{array}{l}16 \\
\text { nd } \\
\text { nd }\end{array}$ \\
\hline $\begin{array}{l}\text { Activités de commerce } \\
\text { vendre des biens et des services (ventes aux enchères par exemple) } \\
\text { commander par l'internet des biens ou services pour son usage personnel }\end{array}$ & $\begin{array}{l}10 \\
28\end{array}$ & $\begin{array}{l}12 \\
32\end{array}$ \\
\hline
\end{tabular}

musique, presse, radio et télévision en ligne), recours croissant aux services relatifs aux voyages et, plus encore, aux services bancaires. Encore en retrait par rapport à la moyenne européenne en 2006, la France a rattrapé son retard, particulièrement pour les activités de communication, les services bancaires, les services liés aux voyages.

En 2006, 9 \% seulement des Français et $18 \%$ des Européens avaient utilisé l'internet au cours des trois derniers mois pour lire et télécharger jeux, images, films ou musique, tandis qu'en 2009, plus d'un quart des Français (26\%) et des Européens (25\%) ont pratiqué cette activité. De même, quand $10 \%$ des Français et $11 \%$ des Européens avaient écouté la radio ou la télévision sur le web en 2006 , ils sont respectivement $25 \%$ et $24 \%$ à l'avoir fait en 2009.

Enfin, près d'un tiers des Français (32\%) ont acheté ou commandé des biens et services pour leur usage privé par l'intermédiaire de l'internet au cours du dernier trimestre, et ils sont $45 \%$ à l'avoir fait au cours des douze derniers mois.

\section{Nette progression du commerce en ligne auprès des particuliers : un Français sur quatre y a recours}

Depuis 2006, le commerce en ligne pour usage personnel a fortement progressé en Europe (+ $42 \%$ dans l'ensemble des 27 États membres) et a concerné, en 2009, $37 \%$ des Européens. Le Royaume-Uni (66\%), le Danemark (64\%), les Pays-Bas et la Suède (63\%) se démarquent dans leur pratique du commerce électronique. La France, en $8^{\mathrm{e}}$ position, est au-dessus de la moyenne européenne (45\%).

Les consommateurs français ont particulièrement choisi de faire leurs achats par l'internet : la progression d'e-commerce en France entre 2006 et 2009 est parmi les plus fortes de l'Union européenne ; elle a doublé pour atteindre $45 \%$ en 2009 (graphique 6).

\section{Graphique 6 - Évolution des commandes réalisées par l'internet, Union européenne des 27 et France, 2006-2009}

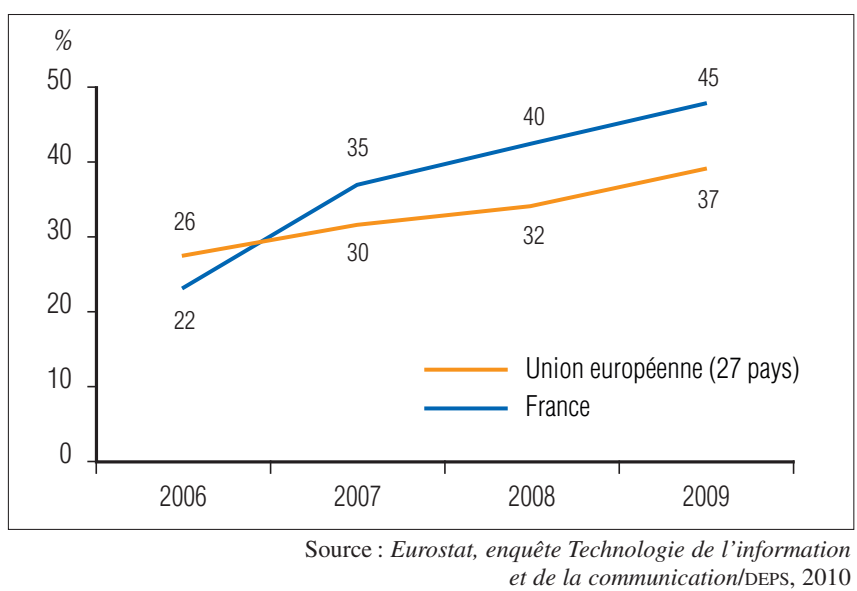




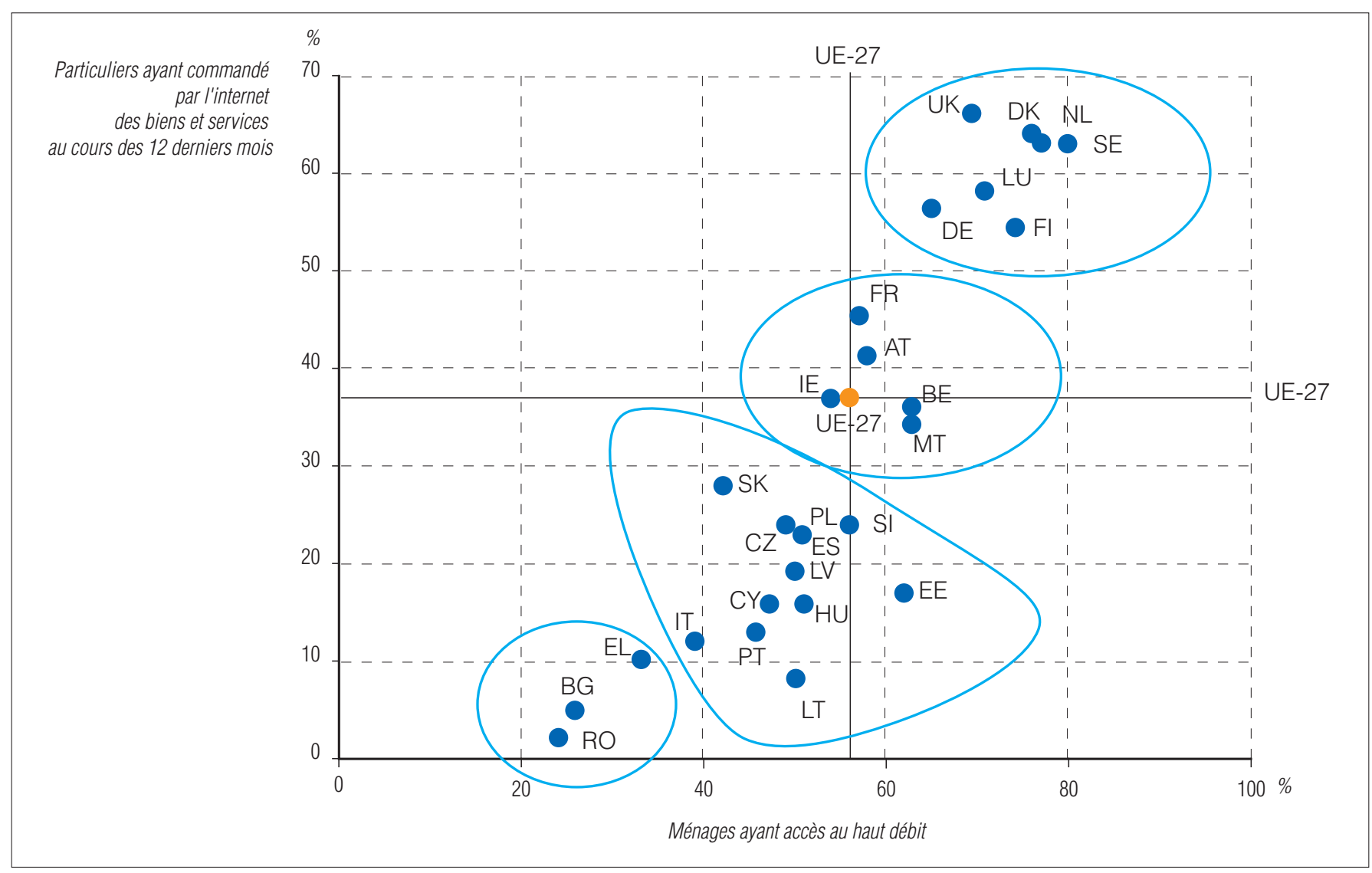

Source : Eurostat, enquête Technologie de l'information et de la communication/DEPS, 2010

Le recours au commerce en ligne est corrélé aux capacités de connexion des particuliers : si l'on rapproche le taux de connexions au haut débit et la part des commandes réalisées par l'intermédiaire de l'internet au cours de l'année 2009, quatre groupes de pays se distinguent (graphique 7).

Le premier groupe est composé des pays dont l'équipement des ménages ne s'est développé qu'au début des années 2000. Ces États sont situés au Sud-Est de l'Union européenne : Roumanie, Bulgarie et Grèce forment cet ensemble pour lequel l'accès à l'internet reste inférieur à $40 \%$ et le taux de connexions à l'internet à haut débit inférieur à $30 \%$. Ce groupe se caractérise par une pratique émergente du commerce en ligne, inférieure à $10 \%$.

Un deuxième groupe rassemble des pays du $\mathrm{Sud}$, du Centre et de la Baltique, assez proches en termes d'équipement numérique (accès à l'internet compris entre $45 \%$ et $60 \%$ et accès au haut débit entre $30 \%$ et $55 \%$ ). Ces pays consomment faiblement par l'intermédiaire de l'internet: leur taux de commandes reste inférieur à $30 \%$. Trois pays ont des comportements spécifiques : la Lettonie, et plus encore l'Estonie, ont des taux d'équipement numérique élevés et, dans le cas de l'Estonie, supérieurs par exemple à ceux de la France, mais des taux de commerce électronique faibles. La Slovaquie, a contrario, se distingue par un accès au haut débit relativement bas (42\%) mais une proportion de commerce électronique se rapprochant de la moyenne européenne (presque $30 \%$ ).

Le troisième groupe réunit les quatre pays proches des moyennes européennes en termes d'équipement et de com- portement d'achat par l'internet: la France, l'Autriche, l'Irlande, la Belgique et Malte.

Enfin, le dernier groupe rassemble les pays où les ménages sont les mieux équipés en termes d'équipement numérique et de haut débit et qui ont largement adopté la consommation par l'internet: de $58 \%$ à $64 \%$ de leurs populations y ont eu recours en 2009.

On constate un lien évident entre le taux d'équipement numérique, le développement du commerce électronique et le niveau général de développement économique, élevé (tableau 6) : Luxembourg, Pays-Bas, Danemark, Suède, Finlande, Royaume-Uni et Allemagne illustrent ce trait. La France se caractérise par des positionnements intermédiaires, que ce soit en termes de développement ou de pratiques (tableau 6).

\section{Les non-consommateurs en ligne préfèrent voir les produits et être fidèles à leurs commerçants}

En 2009, près d'un tiers des Européens n'ont rien acheté par l'intermédiaire de l'internet dans l'année. Les habitants des pays Baltes sont les plus réticents au commerce en ligne : un Estonien sur deux et un Lituanien sur deux n'ont pas acheté par ce canal de vente en 2009.

Les Français ont un comportement proche de la moyenne européenne : 27 Français sur 100 n'ont jamais acheté par l'internet au cours des douze derniers mois. Le premier motif évoqué par les non-consommateurs fran- 
Tableau 6-PIB, accès à l'internet et e-commerce, 27 pays de l'Union européenne, 2009

\begin{tabular}{|c|c|c|c|}
\hline & $\begin{array}{c}\text { PIB } \\
\text { par habitant } \\
\text { en } 2008\end{array}$ & $\begin{array}{c}\text { Accès à } \\
\text { l'internet } \\
\text { des ménages } \\
\text { en } 2009 \\
(\%)\end{array}$ & $\begin{array}{c}\text { Commandes } \\
\text { des } \\
\text { particuliers } \\
\text { en } 2009 \\
(\%)\end{array}$ \\
\hline Union européenne & 100,0 & 65 & 37 \\
\hline Luxembourg & 276,3 & 87 & 58 \\
\hline Irlande & 135,4 & 67 & 37 \\
\hline Pays-Bas & 134,0 & 90 & 63 \\
\hline Danemark & 120,1 & 83 & 64 \\
\hline Suède & 120,0 & 86 & 63 \\
\hline Finlande & 116,8 & 78 & 54 \\
\hline Royaume-Uni & 116,2 & 77 & 66 \\
\hline Allemagne & 115,6 & 79 & 56 \\
\hline Belgique & 115,1 & 67 & 36 \\
\hline France & 107,9 & 63 & 45 \\
\hline Espagne & 102,6 & 54 & 23 \\
\hline Italie & 102,0 & 53 & 12 \\
\hline Chypre & 95,8 & 53 & 16 \\
\hline Grèce & 94,3 & 38 & 10 \\
\hline Slovénie & 90,9 & 64 & 24 \\
\hline République tchèque & 80,3 & 54 & 24 \\
\hline Malte & 76,3 & 64 & 34 \\
\hline Portugal & 76,0 & 48 & 13 \\
\hline Slovaquie & 72,2 & 62 & 28 \\
\hline Estonie & 67,4 & 63 & 17 \\
\hline Hongrie & 64,4 & 55 & 16 \\
\hline Lituanie & 61,9 & 60 & 8 \\
\hline Lettonie & 57,3 & 58 & 19 \\
\hline Pologne & 56,4 & 59 & 23 \\
\hline Bulgarie & 41,3 & 30 & 5 \\
\hline Autriche & nd & 70 & 41 \\
\hline Roumanie & nd & 38 & 2 \\
\hline \multicolumn{4}{|l|}{$\overline{\mathrm{nd} \text { : non disponible }}$} \\
\hline \multicolumn{4}{|c|}{$\begin{array}{l}\text { Note : PIB par habitant en standards de pouvoir d'achat }(\text { SPA })(E U-27=100) \text {. } \\
\text { Commandes: particuliers ayant commandé des biens ou des services sur } \\
\text { l'internet pour leur usage privé au cours de l'année. }\end{array}$} \\
\hline
\end{tabular}

çais est le même que celui mis en avant dans quatorze autres États membres : l'absence de contact avec le produit et avec le commerçant (" préférence pour acheter en personne et voir le produit par habitude ou par fidélité à des magasins »), pour $28 \%$ des consommateurs français et pour $46 \%$ des Estoniens.

La sécurité des transactions financières sur l'internet est un autre motif de préférence pour le commerce physique chez les Français (21\%), les Slovènes (28\%), les Portugais $(21 \%)$ et les Hongrois (23\%). Enfin, l'absence de besoin motive également l'absence de consommation sur l'internet, un motif particulièrement évoqué en Estonie (53\%), Lettonie (40\%) et en Lituanie (48\%).

En Europe comme en France, les jeunes (16 à 24 ans) et les adultes âgés de 35 à 44 ans sont les plus nombreux à n'avoir effectué aucun achat sur l'internet au cours des douze derniers mois, pour les mêmes raisons que l'ensemble des consommateurs: la préférence pour l'achat en magasin, l'absence de besoin d'achat et de sécurité de paiement.

\section{Livres, magazines et billets de spectacle en tête des produits culturels achetés en ligne}

En 2008, la part du chiffre d'affaires des entreprises ${ }^{2}$ réalisée par commerce électronique est de $12 \%$ dans l'Union européenne: $13 \%$ en France contre $28 \%$ en Irlande. En France, les industries alimentaires et d'imprimerie réalisent $26 \%$ de leur chiffre d'affaires par l'internet, les agences de voyages et les services de réservation, $16 \%$.

Les acheteurs en ligne ont privilégié, depuis 2008, les projets de vacances, les voyages et l'hébergement, qui ont progressé de $+20 \%$ dans l'ensemble des 27 États membres, et jusqu'à $+50 \%$ en France. L'achat de logiciels, dont les jeux vidéo, est également en net développement : $29 \%$ des Français en ont commandé en ligne en 2009.

Les produits culturels se prêtent particulièrement bien à l'achat en ligne : $35 \%$ des consommateurs français achètent des livres, magazines et du matériel d'apprentissage ; $33 \%$ réservent des spectacles en ligne ; $27 \%$ commandent des films et de la musique (tableau 7).

\section{Le niveau d'éducation et le sexe influent sur la consommation en ligne}

La cartographie des consommateurs internautes français révèle des différences entre grandes régions : le NordPas-de-Calais et l'Ouest concentrent les plus faibles adeptes du commerce électronique, avec respectivement $28 \%$ et $33 \%$ d'achats pour usage personnel de biens ou services au cours de l'année 2009. À l'opposé, les consommateurs d'Île-de-France $(49 \%)$ et de la Méditerranée (48\%) ont des habitudes d'achat par l'internet légèrement supérieures à la moyenne française $(45 \%)$.

Bien plus que la densité des zones de résidence, le niveau d'éducation des consommateurs semble être un facteur influant sur le choix des habitudes de consommation physique ou en ligne, dans l'Union européenne comme en France, où l'écart entre les particuliers ayant reçu une éducation scolaire formelle de base ${ }^{3}$ et ceux titulaires d'un diplôme de l'enseignement supérieur est de 40 points.

2. Tous secteurs d'activités sauf secteur financier. Concerne les entreprises de 10 personnes employées ou plus.

3. Dans la nomenclature choisie par Eurostat, les niveaux d'éducation sont regroupés en trois grandes catégories :

- l'éducation scolaire «formelle de base» inclut l'éducation maternelle, l'enseignement primaire et le premier cycle de l'enseignement secondaire ;

- l'éducation scolaire «formelle moyenne » inclut le deuxième cycle de l'enseignement secondaire, l'enseignement postsecondaire qui n'est pas du supérieur ;

- l'éducation scolaire «formelle élevée » inclut l'enseignement supérieur. 
Tableau 7 - Commandes des particuliers pour leur usage privé par l'intermédiaire de l'internet au cours des 12 derniers mois, Union européenne des 27 et France, 2006-2009

\begin{tabular}{|c|c|c|c|c|c|c|c|c|}
\hline & \multicolumn{4}{|c|}{ Union européenne (27 pays) } & \multicolumn{4}{|c|}{ France } \\
\hline & 2006 & 2007 & 2008 & 2009 & 2006 & 2007 & 2008 & 2009 \\
\hline \multicolumn{9}{|l|}{ Produits et services culturels } \\
\hline livres, magazines, matériel d'apprentissage & 39 & 38 & 39 & 33 & 25 & 39 & 34 & 35 \\
\hline billets de spectacle & 30 & 31 & 33 & 35 & 24 & 30 & 33 & 33 \\
\hline films, musique & 34 & 33 & 29 & 32 & 26 & 30 & 27 & 27 \\
\hline appareils électroniques (y compris appareils photo) & 24 & 24 & 25 & 26 & 7 & 19 & 14 & 20 \\
\hline \multicolumn{9}{|l|}{ Produits et services non culturels } \\
\hline voyages et hébergements de vacances & 41 & 43 & 42 & 51 & 35 & 41 & 44 & 67 \\
\hline vêtements ou articles de sport & 36 & 39 & 41 & 46 & 34 & 49 & 47 & 53 \\
\hline articles domestiques (meubles, jouets, etc.) & 30 & 34 & 35 & 37 & 27 & 41 & 40 & 39 \\
\hline logiciels (y compris jeux vidéo) & 25 & 24 & 21 & 29 & 13 & 17 & 15 & 29 \\
\hline matériel informatique & 19 & 17 & 16 & 18 & 22 & 26 & 24 & 26 \\
\hline autres types de biens ou de services & 12 & 9 & 8 & 9 & 11 & 10 & 10 & 10 \\
\hline denrées alimentaires & 10 & 10 & 11 & 13 & 5 & 6 & 11 & 8 \\
\hline actions, services financiers, assurance & 11 & 9 & 9 & 10 & 3 & 6 & 5 & 4 \\
\hline jeux de hasard ou paris & 6 & 6 & 7 & nd & nd & 5 & 3 & nd \\
\hline
\end{tabular}

Source : Eurostat, enquête Technologie de l'information et de la communication/DEPS, 2010

À l'échelon européen, les particuliers possédant une éducation scolaire de base sont $18 \%$ à avoir commandé par l'internet au cours de l'année 2009, contre $61 \%$ d'internautes consommateurs diplômés de l'enseignement supérieur. En France, $29 \%$ des personnes ayant été scolarisées jusqu'à la fin du collège ont consommé sur l'internet, contre $72 \%$ de personnes diplômées du supérieur (graphique 8 ).

En général, les hommes achètent plus par l'internet que les femmes : en 2009, $40 \%$ des hommes de l'Union européenne âgés de 16 à 74 ans ont commandé par l'internet pour leur usage personnel, contre $34 \%$ de femmes. En France, ces taux s'élèvent à $46 \%$ et $43 \%$, la proportion des hommes étant légèrement supérieure à la moyenne européenne et celle des femmes légèrement inférieure. Les femmes d'un niveau scolaire de base consomment moins par l'internet que les hommes.

En revanche, le sexe n'est pas un critère discriminant parmi les personnes diplômées puisque $72 \%$ de femmes et autant d'hommes ont commandé par l'internet en 2009. En 2008, la proportion de femmes était même supérieure à celle des hommes (67\% contre $65 \%)$.

\section{Graphique 8 - Particuliers ayant commandé des biens ou des services sur l'internet au cours de l'année, par niveau d'éducation, Union européenne des 27 et France, 2006-2009}

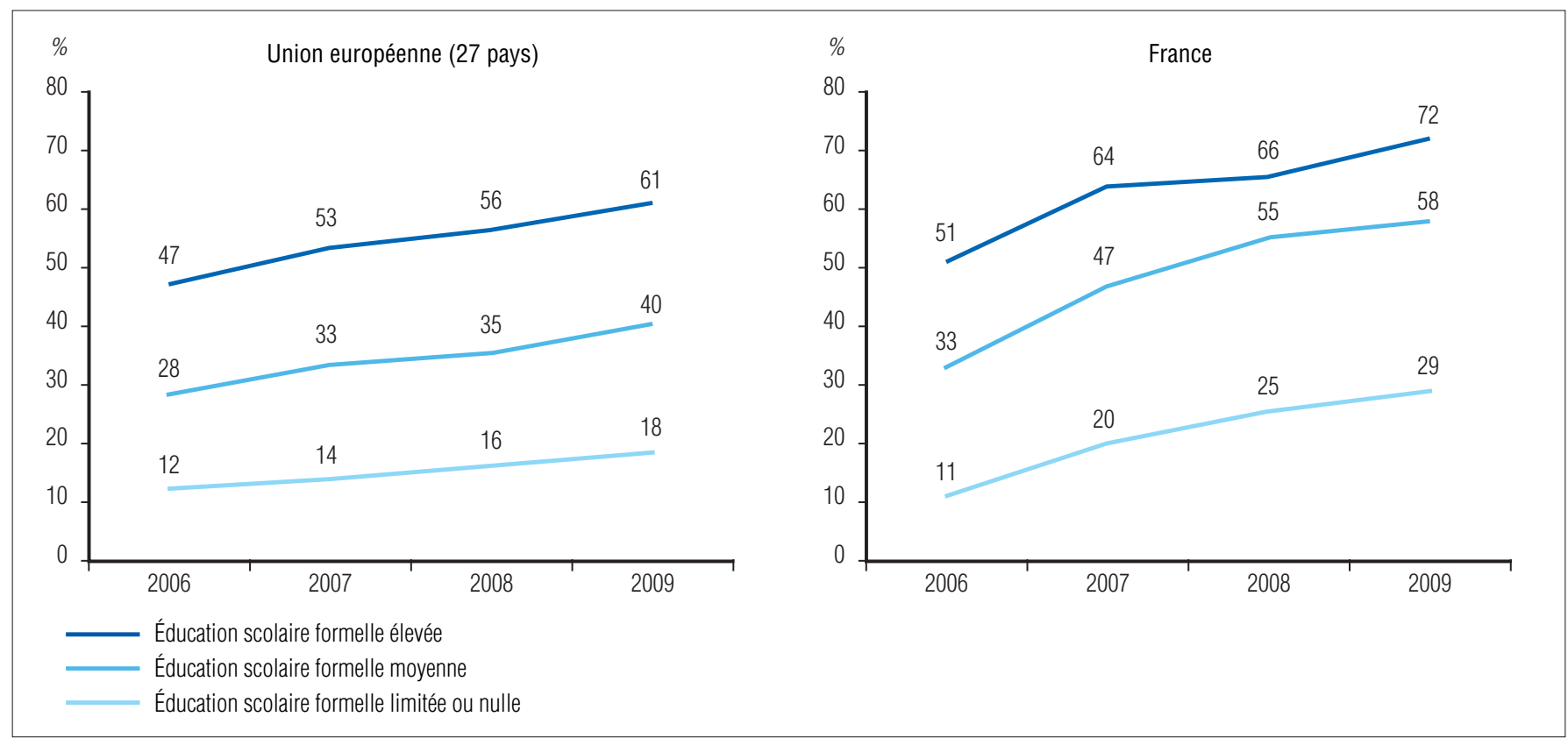


Graphique 9 - Commandes par l'internet de biens ou services pour usage privé, par origine des vendeurs, 27 pays de l'Union européenne, 2009

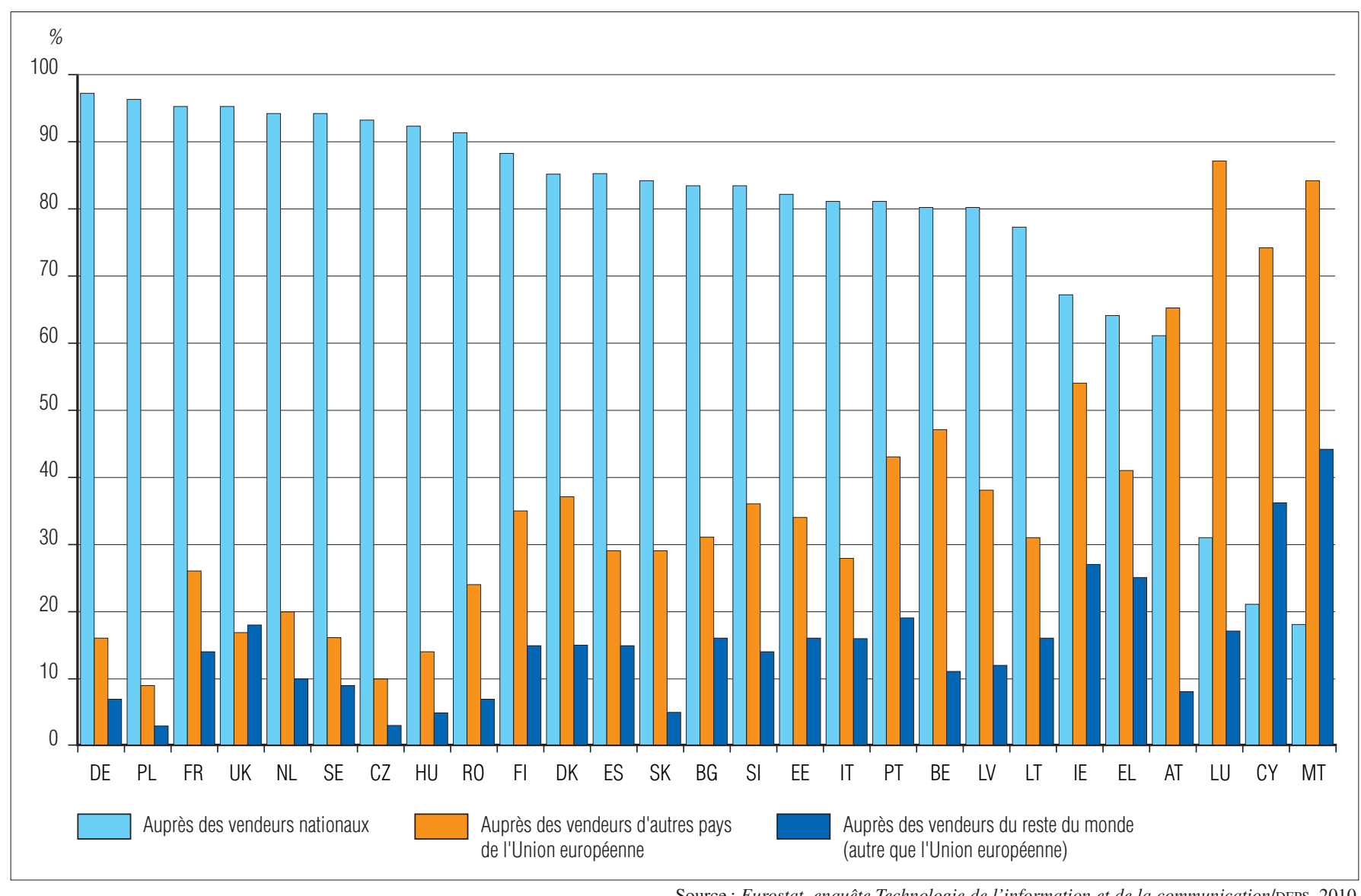

Enfin, à l'exception du Luxembourg, de Malte, de Chypre et de l'Autriche, les consommateurs de l'Union européenne ont privilégié des vendeurs nationaux au cours de l'année 2009. Les particuliers français sont $95 \%$ à avoir commandé des biens ou services par l'internet auprès de vendeurs nationaux au cours des douze derniers mois; $26 \%$ auprès des vendeurs d'autres pays de l'Union européenne et $14 \%$ auprès de vendeurs du reste du monde (autres que l'Union européenne) (graphique 9). 


\section{Éléments de méthodologie}

L'enquête communautaire sur les technologies de l'information et de la communication (TIC ou, en anglais, ICT "Information and Communication Technologies") réalisée auprès des ménages a pour objectif de collecter des informations:

- décrivant l'équipement et les usages des ménages dans le domaine des technologies de l'information et de la communication (informatique, dispositifs d'accès à l'internet et au haut débit, téléphonie);

- concernant les fréquences d'utilisation, les compétences en la matière ainsi que les obstacles au recours aux nouvelles technologies, les achats en ligne, les services en ligne, la sécurité.

Cette enquête s'inscrit dans le cadre du règlement n 808/2004 adopté le 21 avril 2004 par le Parlement européen et le Conseil de l'Union européenne concernant les statistiques communautaires sur la société de l'information.

Les résultats présentés sont extraits de la base de données d'Eurostat, l'office statistique de la Commission europénne chargé de fournir des statistiques permettant des comparaisons entre les États et les régions (http://ec.europa.eu/eurostat). En France, l'enquête est réalisée par l'Institut national de la statistique et des études économiques (Insee).

Le champ communautaire couvre les ménages comptant au moins une personne âgée de 16 à 74 ans et les particuliers âgés de 16 à 74 ans : en 2009, l'échantillon de l'Union européenne des 27 contenait 161140 ménages et 209843 individus.

Depuis 2002, l'enquête est de périodicité annuelle; la période de référence est le premier trimestre de l'année courante. De plus elle évalue chaque année des thèmes spécifiques : en 2008, elle concernait les usages; en 2009, les achats en ligne : les particuliers étaient interrogés sur leurs achats de biens ou services en ligne pour usage personnel au cours des douze derniers mois précédant l'enquête.

Code des pays:

$\begin{array}{ll}\text { EU } 27 & \text { Union européenne (27 pays) } \\ \text { AT } & \text { Autriche } \\ \text { BE } & \text { Belgique } \\ \text { BG } & \text { Bulgarie } \\ \text { CY } & \text { Chypre } \\ \text { CZ } & \text { République tchèque } \\ \text { DE } & \text { Allemagne (incluant l'ex-RDA } \\ & \text { à partir de 1991) } \\ \text { DK } & \text { Danemark } \\ \text { EE } & \text { Estonie }\end{array}$

$\begin{array}{ll}\text { EL } & \text { Grèce } \\ \text { ES } & \text { Espagne } \\ \text { FI } & \text { Finlande } \\ \text { FR } & \text { France } \\ \text { HU } & \text { Hongrie } \\ \text { IE } & \text { Irlande } \\ \text { IT } & \text { Italie } \\ \text { LT } & \text { Lituanie } \\ \text { LU } & \text { Luxembourg (Grand-Duché) } \\ \text { LV } & \text { Lettonie }\end{array}$

MT Malte

NL Pays-Bas

$\mathrm{PL} \quad$ Pologne

PT Portugal

RO Roumanie

SE Suède

SI Slovénie

SK Slovaquie

UK Royaume-Uni

\section{Définitions de quelques termes techniques}

Technologies de l'information et de la communication (TIC) : l'acronyme qui désigne les technologies de l'information et de la communication est un concept vaste qui englobe non seulement les équipements et les techniques utilisés dans le traitement et la transmission des informations, que ce soit dans les domaines de l'informatique, de l'internet, de la télévision et des télécommunications, mais aussi les usages qui se développent autour de l'utilisation de ces nouveaux supports. Par extension, on parle aussi de société de l'information.

Ordinateur de bureau : ordinateur avec clavier et écran qui demeure dans un endroit déterminé.

Ordinateur portable (laptop) : ordinateur assez petit pour être transporté et capable d'effectuer les mêmes tâches qu'un ordinateur de bureau. Les ordinateurs de poche, bien que portables, n'en font pas partie.

Ordinateur «à main " ou « de poche " (palmtop ou PDA pour Personal digital assistant) : ordinateur ou assistant numérique personnel qui peut être utilisé tenu dans la main et stocké dans un étui ou une poche.

Modem (abréviation de modulateur-démodulateur) : appareil servant à traduire des informations numériques en signaux analogiques, et inversement.

Haut débit ou «à large bande » : caractérise une liaison permettant de transmettre de grandes quantités de données. Toute connexion dont le débit (ascendant) est de 128 ko (kilo-octet) au minimum sera considérée comme du haut débit.

DsL (Digital Subscriber Line) : ligne d'accès qui permet un débit de transmission des signaux numériques supérieur à celui d'un modem à fréquence vocale (débit supérieur à $144 \mathrm{kbit} / \mathrm{seconde}$ ).

AdsL (Asymmetrical Digital Subscriber Line) : I'ADSL permet de transporter plusieurs mégabits par seconde sur les fréquences hautes des deux fils de cuivre du téléphone. Les données peuvent être transmises jusqu'à $20 \mathrm{Mbits} / \mathrm{seconde}$ en téléchargement.

SDSL (Symmetric Digital Subscriber Line) : la SDSL est une technique qui, contrairement à la ligne ADSL, a des débits symétriques : son débit en réception (download) est égal au débit en envoi (upload).

Autre connexion à large bande : tout accès haut débit à l'internet qui n'utilise pas les technologies DSL (exemples: le câble modem, le réseau Lan - Local area network -, la technologie FWA - Fixed Wireless Access).

Accès sans fil à l'internet : accès à l'internet par des moyens de radiocommunication, par exemple selon les normes internationales dites Wifi (abréviation de l'anglais wireless fidelity) et Wimax (Worldwide Interoperability for Microwave Access). 
Clé 3G : troisième génération de téléphones basée sur l'UMTs.

WAP (Wireless Application Protocol) : protocole de communication qui permet d'accéder à l'internet à partir d'un appareil sans fil, par exemple un téléphone portable ou un assistant personnel.

Gprs (Global Packet Radio Services) : support de transmission de données entre un téléphone portable 2G et l'internet. Le débit de transmission peut atteindre $115 \mathrm{kbit} / \mathrm{seconde}$.

UmTs: Universal Mobile Telecommunications System. Système de téléphonie mobile de troisième génération (3G). Elle permet des débits numériques jusqu'à 2 Mbits/seconde.

Mesures informatiques : en informatique, l'unité de base est le bit (binary digit), 8 bits forment un octet. $1 \mathrm{~kg}$-octet $(\mathrm{ko})=10^{3}=1024$ octets ; 1 méga-octet $(\mathrm{Mo})=10^{6}=1048576$ octets $; 1$ giga-octet $(\mathrm{Go})=10^{9}=1073741824$ octets. Le kilobit par seconde (kbit/s) est une unité permettant de mesurer la vitesse de transfert de données.

\section{RÉSUMÉ}

Issue de l'enquête européenne « TIC - Technologies de l'information et de la communication », l'exploitation des données pour la France permet de comparer l'équipement informatique et les usages numériques des Français par rapport à leurs voisins européens. Si l'utilisation quotidienne d'un ordinateur et l'accès à l'internet concernent désormais plus d'un Français sur deux, l'âge et le sexe restent des critères discriminants pour l'usage des nouvelles technologies : les hommes y ont plus recours que les femmes, les jeunes plus que leurs aînés. La communication (messagerie) et les loisirs culturels font partie des activités préférées des usagers de l'internet, tandis que le commerce en ligne et les services, notamment bancaires, se développent depuis 2006. Le déploiement des pratiques nomades est encore émergent. Au plan européen, l'écart est net entre les pays du Nord, fortement équipés et familiers des usages numériques, et les pays du Sud, où l'équipement numérique est plus récent.

\section{ABSTRACT}

Data from the European "ICT: Information and Communications Technologies" survey provides an opportunity to compare France's computer equipment ownership and digital activity levels with those of its European neighbours. Although over 50\% of French people make daily use of computers and access the internet, age and sex remain differentiating factors in new technology usage, with men making more use of it than women, and younger people more than older people. Communications (messaging) and cultural leisure activities are amongst internet users'favourite activities, whilst online trading and services, particularly online banking, have been on the increase since 2006. The spread of nomadic practices is still gaining momentum. At European level, there is a marked disparity between northern European countries, with their high levels of computing competence and ownership, and southern Europe where the adoption of computer equipment is more recent.

Tous les documents publiés par le DEPS sont téléchargeables sur http://www.culture.gouv.fr/deps

Le DEPS n'assurant pas de diffusion physique de ces documents, nous vous proposons de vous informer régulièrement des parutions par message électronique. Pour ce faire, merci de bien vouloir nous communiquer votre courriel à l'adresse contactdeps.ddai@culture.gouv.fr 